\title{
Persistence of frequently transmitted drug-resistant HIV-1 variants can be explained by high viral replication capacity
}

Marieke Pingen ${ }^{1,2}$, Annemarie MJ Wensing ${ }^{2}$, Katrien Fransen ${ }^{3}$, Annelies De Bel ${ }^{4}$, Dorien de Jong ${ }^{2}$, Andy IM Hoepelman ${ }^{5}$, Emmanouil Magiorkinis ${ }^{6}$, Dimitrios Paraskevis ${ }^{6}$, Maja M Lunar ${ }^{7}$, Mario Poljak Monique Nijhuis ${ }^{2}$, Charles AB Boucher ${ }^{1 *}$ and On behalf of the SPREAD programme

\begin{abstract}
Background: In approximately 10\% of newly diagnosed individuals in Europe, HIV-1 variants harboring transmitted drug resistance mutations (TDRM) are detected. For some TDRM it has been shown that they revert to wild type while other mutations persist in the absence of therapy. To understand the mechanisms explaining persistence we investigated the in vivo evolution of frequently transmitted HIV-1 variants and their impact on in vitro replicative capacity.

Results: We selected 31 individuals infected with HIV-1 harboring frequently observed TDRM such as M41L or $\mathrm{K} 103 \mathrm{~N}$ in reverse transcriptase (RT) or M46L in protease. In all these samples, polymorphisms at non-TDRM positions were present at baseline (median protease: 5, RT: 6). Extensive analysis of viral evolution of protease and RT demonstrated that the majority of TDRM (51/55) persisted for at least a year and even up to eight years in the plasma. During follow-up only limited selection of additional polymorphisms was observed (median: 1).

To investigate the impact of frequently observed TDRM on the replication capacity, mutant viruses were constructed with the most frequently encountered TDRM as site-directed mutants in the genetic background of the lab strain HXB2. In addition, viruses containing patient-derived protease or RT harboring similar TDRM were made. The replicative capacity of all viral variants was determined by infecting peripheral blood mononuclear cells and subsequently monitoring virus replication. The majority of site-directed mutations (M46I/M46L in protease and M41L, M41L + T215Y and $\mathrm{K} 103 \mathrm{~N}$ in RT) decreased viral replicative capacity; only protease mutation L90M did not hamper viral replication. Interestingly, most patient-derived viruses had a higher in vitro replicative capacity than the corresponding site-directed mutant viruses.
\end{abstract}

Conclusions: We demonstrate limited in vivo evolution of protease and RT harbouring frequently observed TDRM in the plasma. This is in line with the high in vitro replication capacity of patient-derived viruses harbouring TDRM compared to site-directed mutant viruses harbouring TDRM. As site-directed mutant viruses have a lower replication capacity than the patient-derived viruses with similar mutational patterns, we propose that (baseline) polymorphisms function as compensatory mutations improving viral replication capacity.

Keywords: HIV, Drug resistance, Transmission, Evolution, Reversion, Persistence, Compensatory fixation

\footnotetext{
*Correspondence: c.boucher@erasmusmc.nl

'Department of Virology, Viroscience Lab, Erasmus MC, Postbus 2040, 3000

CA Rotterdam, the Netherlands

Full list of author information is available at the end of the article
} 


\section{Background}

The viral enzymes reverse transcriptase (RT) and protease were the first targets of antiretroviral therapy and the most commonly used drug regimens still aim at inhibiting these viral proteins [1]. In resource-rich settings, drug resistance mutations in protease and RT are detected in $10-15 \%$ of newly diagnosed HIV patients $[2,3]$.

The majority of transmitted drug-resistant viruses contain limited resistance profiles to single drug classes. Nucleoside RT inhibitor (NRTI) mutations are the most frequently observed transmitted drug resistance mutations (TDRM). Especially thymidine analogue mutations (TAMs) M41L and T215 variants, that have been selected by drugs extensively used in the past, are often observed in newly diagnosed patients [4]. A worrying trend is the increased prevalence of non-nucleoside RT inhibitor (NNRTI) related mutations in newly diagnosed patients $[3,5]$, as single NNRTI mutations, such as the frequently observed K103N mutation, can result in high levels of resistance against first generation NNRTIs [6]. In protease, M46I/L and L90M are the most frequently observed TDRM $[2,3]$. When present in combination with other protease drug resistance mutations, both M46I/L and L90M are related to reduced susceptibility to several protease inhibitors (PIs) [6].

It is generally acknowledged that most drug resistance mutations decrease the replicative capacity (RC) of HIV$1[7,8]$. As such, in the absence of drugs TDRM can revert to wild type, thereby increasing viral RC. Indeed, follow-up of untreated individuals diagnosed with a drug resistant HIV variant revealed that certain mutations with a detrimental effect on the viral RC, such as M184V in $\mathrm{RT}$, after transmission to a new host often revert rapidly in the plasma $[9,10]$. In addition, the use of very sensitive assays shows that minority drug resistance mutations are frequently found in untreated individuals, suggestive of reversion after transmission $[11,12]$.

However, follow-up of patients diagnosed with HIV-1 harboring TDRM has revealed that TAMs, PI- and NNRTI-related TDRM often persist for prolonged periods [10,13-25]. The mechanisms explaining persistence have not been fully resolved. Based on the available literature [13,15-25], we have previously proposed two possible mechanisms to explain persistence of TDRM [9]. When the effect of the TDRM on the RC is very small, reversion may take a very long time. Alternatively, when the TDRM decreases the RC considerably the presence or selection of additional compensatory mutations can prevent reversion of the TDRM.

The aim of our study was to gain more insight in the mechanisms causing persistence of drug resistant HIV-1 variants after transmission. Therefore, we investigated the molecular evolution of HIV-1 protease and RT harboring the most frequently observed TDRM in great detail. The majority of TDRM persisted during the followup, and only few additional polymorphisms were selected during this period. Most patient-derived viruses had a higher RC than the corresponding site-directed mutant viruses, indicating that persistence can be explained by a high replication capacity of most transmitted drug resistant HIV-1 variants.

\section{Results}

\section{Patients diagnosed with a transmitted drug resistant} HIV-1 variant

To investigate the in vivo evolution of transmitted drug resistant HIV variants, we selected 31 patients from four European countries (Belgium, Greece, the Netherlands, Slovenia) who were diagnosed in 2001 to 2008 with an HIV variant harboring a frequently observed TDRM (prevalence $>5 \%$ in patients diagnosed with HIV-1 harboring TDRM in the SPREAD-programme). Patients were included if a plasma sample was available at one year (10-14 months) after diagnosis if therapy was not yet initiated. If available, a third time point before start of treatment was investigated. Prior negative HIV tests were available for 14 patients, revealing that at least nine patients had been infected for less than two years. The majority of the patients were men having sex with men (MSM), which is the most important route of transmission in Western Europe. The median plasma HIV-RNA in our group of patients was 4.6 log copies/ $\mathrm{ml}$, comparable to the median HIV-RNA observed in the SPREAD-programme in 2002-2006 (4.8 log cop$\mathrm{ies} / \mathrm{ml}$ ). The median baseline CD4 count was 653 cells/ $\mathrm{mm}^{3}$, which is higher than the median observed in the SPREAD programme (343 cells $/ \mathrm{mm}^{3}$ ) [3].

Surveillance studies demonstrated that most transmitted drug resistant $\mathrm{HIV}-1$ variants harbor resistance against a single drug class $[3,4]$. In line with this observation, only $3 / 31$ of the patients selected for this study had been diagnosed with an HIV-1 variant resistant to multiple drug classes. A total of 55 mutations at positions included in the WHO list for surveillance of transmitted drug resistant HIV-1 [26] were observed in the transmitted viruses at baseline. A single TDRM was detected in 10/16 patients with viruses harboring only NRTI-related TDRM, for the other six patients a profile of two to four TDRM was observed. The vast majority of NRTI-related TDRM were TAM-related mutations. In six of the selected patients viral variants containing a single NNRTI-related TDRM were observed. Six patients were diagnosed with HIV-1 harboring a single PI-related TDRM (Table 1). In addition to TDRM, polymorphisms were present in all baseline sequences. For variants containing RT TDRM, the median number of RT polymorphisms was 7 (range: 4-21) when compared to HXB2 and 6 to consensus B (range: 2-19). Viruses harboring PR resistance mutations had a median 
Table 1 Patient characteristics, resistance mutations and evolution

\begin{tabular}{|c|c|c|c|c|c|c|c|c|c|c|c|c|c|}
\hline ID & Gender & $\begin{array}{l}\text { Last } \\
\text { negative } \\
\text { HIV test }\end{array}$ & $\begin{array}{l}\text { Country } \\
\text { of origin }\end{array}$ & Diagnosis & $\begin{array}{l}\text { Risk } \\
\text { group }\end{array}$ & $\begin{array}{l}\text { Months } \\
\text { after first } \\
\text { analysis }\end{array}$ & $\begin{array}{l}\text { Plasma } \\
\text { HIV RNA } \\
\text { (copies/ml) }\end{array}$ & $\begin{array}{l}\text { CD4 } \\
\text { count }\end{array}$ & $\begin{array}{l}\text { Sub- } \\
\text { type }\end{array}$ & $\begin{array}{l}\text { Resistance } \\
\text { Profile PR }\end{array}$ & $\begin{array}{l}\text { Resistance } \\
\text { Profile RT }\end{array}$ & p-distance & $\begin{array}{l}\text { p-value } \\
\text { dN/dS }\end{array}$ \\
\hline \multicolumn{14}{|c|}{ Transmitted variants harboring only NRTI-related mutations } \\
\hline \multirow[t]{3}{*}{ P01 } & Male & & $\mathrm{NL}$ & May 2007 & MSM & 0 & $>750000$ & 461 & B & & M41L & & \\
\hline & & & & & & 10 & & & & & M41L & 0.001 & 1.000 \\
\hline & & & & & & 16 & & & & & M41L & 0.002 & 0.290 \\
\hline \multirow[t]{3}{*}{ P02 } & Male & & $\mathrm{NL}$ & Jan 2008 & MSM & 0 & 21800 & 423 & B & & M41L & & \\
\hline & & & & & & 12 & & & & & M41L & 0.005 & 0.225 \\
\hline & & & & & & 28 & & & & & M41L & 0.005 & 0.225 \\
\hline \multirow[t]{3}{*}{ P03 } & Male & Jan 2004 & $\mathrm{BE}$ & Jun 2005 & MSM & 0 & 41000 & 483 & B & & M41L & & \\
\hline & & & & & & 11 & & & & & M41L & 0.000 & 1.000 \\
\hline & & & & & & 32 & & & & & M41L & 0.002 & 0.152 \\
\hline \multirow[t]{3}{*}{ P04 } & Male & & $\mathrm{NL}$ & Feb 2007 & MSM & 0 & 102000 & 322 & B & & L210LS & & \\
\hline & & & & & & 11 & & & & & - & 0.000 & 1.000 \\
\hline & & & & & & 25 & & & & & - & 0.002 & 1.000 \\
\hline \multirow[t]{3}{*}{ P05 } & Male & & SL & Jun 2001 & MSM & 0 & 12267 & 950 & B & & $\mathrm{T} 215 \mathrm{D}$ & & \\
\hline & & & & & & 12 & & & & & $\mathrm{~T} 215 \mathrm{D}$ & 0.011 & 0.060 \\
\hline & & & & & & 99 & & & & & $\mathrm{~T} 215 \mathrm{D}$ & 0.007 & 0.428 \\
\hline \multirow[t]{3}{*}{ P06 } & Male & Mar 2005 & $S L$ & Feb 2006 & MSM & 0 & 797000 & 953 & B & & T215S & & \\
\hline & & & & & & 14 & & & & & T215S & 0.000 & 1.000 \\
\hline & & & & & & 21 & & & & & T215S & 0.000 & 1.000 \\
\hline \multirow[t]{3}{*}{ P07 } & Male & & $\mathrm{NL}$ & Sep 2008 & MSM & 0 & 36300 & 521 & B & & $\mathrm{T} 215 \mathrm{D}$ & & \\
\hline & & & & & & 11 & & & & & $\mathrm{~T} 215 \mathrm{D}$ & 0.000 & 1.000 \\
\hline & & & & & & 27 & & & & & $\mathrm{~T} 215 \mathrm{D}$ & 0.000 & 1.000 \\
\hline \multirow[t]{2}{*}{ P08 } & Male & Sep 2004 & $\mathrm{NL}$ & Dec 2004 & MSM & 0 & 583000 & 596 & B & & T215IT & & \\
\hline & & & & & & 13 & & & & & - & 0.000 & 1.000 \\
\hline \multirow[t]{3}{*}{ P09 } & Male & Sep 2006 & $\mathrm{NL}$ & Sep 2007 & MSM & 0 & 158000 & 678 & B & & T215AT & & \\
\hline & & & & & & 13 & & & & & T215AT & 0.001 & 0.294 \\
\hline & & & & & & 20 & & & & & $\mathrm{~T} 215 \mathrm{~A}$ & 0.000 & 1.000 \\
\hline \multirow[t]{3}{*}{ P10 } & Male & Oct 2003 & $\mathrm{NL}$ & Jan 2005 & MSM & 0 & 89800 & 289 & B & & K219N & & \\
\hline & & & & & & 11 & & & & & K219N & 0.000 & 1.000 \\
\hline & & & & & & 44 & & & & & K219N & 0.000 & 1.000 \\
\hline \multirow[t]{2}{*}{ P11 } & Male & & $\mathrm{BE}$ & Mar 2006 & HSX & 0 & 318000 & 966 & B & & D67N T215C & & \\
\hline & & & & & & 13 & & & & & D67N T215C & 0.001 & 0.291 \\
\hline \multirow[t]{3}{*}{ P12 } & Male & & $\mathrm{NL}$ & Feb 2007 & MSM & 0 & 55900 & 609 & B & & $\begin{array}{l}\text { D67G T215C } \\
\text { K219E }\end{array}$ & & \\
\hline & & & & & & 11 & & & & & $\begin{array}{l}\text { D67G T215C } \\
\text { K219E }\end{array}$ & 0.007 & 0.156 \\
\hline & & & & & & 24 & & & & & $\begin{array}{l}\text { D67G T215C } \\
\text { K219E }\end{array}$ & 0.000 & 1.000 \\
\hline \multirow[t]{3}{*}{ P13 } & Male & Jul 2004 & $\mathrm{NL}$ & Nov 2007 & MSM & 0 & 294000 & 531 & B & & $\begin{array}{l}\text { D67G T215C } \\
\text { K219E }\end{array}$ & & \\
\hline & & & & & & 12 & & & & & $\begin{array}{l}\text { D67G T215C } \\
\text { K219E }\end{array}$ & 0.000 & 1.000 \\
\hline & & & & & & 14 & & & & & $\begin{array}{l}\text { D67G T215C } \\
\text { K219E }\end{array}$ & 0.000 & 1.000 \\
\hline
\end{tabular}


Table 1 Patient characteristics, resistance mutations and evolution (Continued)

\begin{tabular}{|c|c|c|c|c|c|c|c|c|c|c|c|c|c|}
\hline \multirow[t]{2}{*}{ P14 } & \multirow[t]{2}{*}{ Male } & \multirow[t]{6}{*}{ Apr 2005} & \multirow[t]{2}{*}{$\mathrm{NL}$} & \multirow[t]{2}{*}{ Jun 2005} & \multirow[t]{2}{*}{ HSX } & 0 & \multirow[t]{2}{*}{750000} & \multirow[t]{2}{*}{577} & \multirow{2}{*}{\multicolumn{2}{|c|}{ B }} & \multicolumn{3}{|l|}{$\begin{array}{l}\text { D67G T215C } \\
\text { K219E }\end{array}$} \\
\hline & & & & & & 14 & & & & & $\begin{array}{l}\text { D67G T215C } \\
\text { K219E }\end{array}$ & 0.000 & 1.000 \\
\hline \multirow[t]{4}{*}{ P15 } & \multirow[t]{4}{*}{ Male } & & \multirow[t]{4}{*}{ NL } & \multirow[t]{4}{*}{ Aug 2005} & \multirow[t]{4}{*}{ MSM } & 0 & \multirow[t]{4}{*}{81000} & \multirow[t]{4}{*}{470} & \multirow{4}{*}{\multicolumn{2}{|c|}{ B }} & \multicolumn{2}{|l|}{$\begin{array}{l}\text { M41L T69S } \\
\text { T210E T215ST }\end{array}$} & 1.000 \\
\hline & & & & & & 11 & & & & & $\begin{array}{l}\text { M41L T69S } \\
\text { T210DE T215ST }\end{array}$ & 0.000 & 1.000 \\
\hline & & & & & & 39 & & & & & $\begin{array}{l}\text { M41L T69S } \\
\text { T210E T215ST }\end{array}$ & 0.000 & 1.000 \\
\hline & & & & & & 77 & & & & & $\begin{array}{l}\text { M41L T69S } \\
\text { T210E T215ST }\end{array}$ & 0.000 & 1.000 \\
\hline \multirow[t]{4}{*}{ P16 } & \multirow[t]{4}{*}{ Male } & \multirow[t]{4}{*}{ Mar 2005} & \multirow[t]{4}{*}{$\mathrm{NL}$} & \multirow[t]{4}{*}{ Jun 2006} & MSM & 0 & 34600 & 1129 & B & & $\begin{array}{l}\text { M41L T69S } \\
\text { T210E T215ST }\end{array}$ & & \\
\hline & & & & & & 13 & & & & & $\begin{array}{l}\text { M41L T69AS } \\
\text { T210E T215ST }\end{array}$ & 0.000 & 1.000 \\
\hline & & & & & & 33 & & & & & $\begin{array}{l}\text { M41L T69S } \\
\text { T210E T215ST }\end{array}$ & 0.001 & 1.000 \\
\hline & & & & & & 49 & & & & & $\begin{array}{l}\text { M41L T69S } \\
\text { T210E T215ST }\end{array}$ & 0.001 & 1.000 \\
\hline Tran & smitted & ariants harb & g onl & I-related m & itations & & & & & & & & \\
\hline P17 & Male & Feb 2005 & $\mathrm{NL}$ & Sep 2006 & MSM & 0 & 5990 & 790 & B & & $\mathrm{K} 103 \mathrm{~N}$ & & \\
\hline & & & & & & 12 & & & & & $\mathrm{~K} 103 \mathrm{~N}$ & 0.000 & 1.000 \\
\hline & & & & & & 30 & & & & & $\mathrm{~K} 103 \mathrm{~N}$ & 0.000 & 1.000 \\
\hline P18 & Male & Jun 2004 & $\mathrm{BE}$ & Apr 2006 & MSM & 0 & 39900 & 648 & B & & $\mathrm{K} 103 \mathrm{~N}$ & & \\
\hline & & & & & & 12 & & & & & K103N & 0.000 & 1.000 \\
\hline & & & & & & 28 & & & & & $\mathrm{~K} 103 \mathrm{~N}$ & 0.000 & 1.000 \\
\hline P19 & Male & & $\mathrm{NL}$ & Sep 2005 & MSM & 0 & 21400 & 359 & B & & K103Q & & \\
\hline & & & & & & 12 & & & & & K103Q & 0.000 & 1.000 \\
\hline & & & & & & 59 & & & & & K103Q & 0.001 & 0.304 \\
\hline P20 & Male & 1995 & $\mathrm{SL}$ & Sep 2005 & MSM & 0 & 29300 & 421 & B & & Y181C & & \\
\hline & & & & & & 11 & & & & & Y181C & 0.000 & 1.000 \\
\hline & & & & & & 49 & & & & & Y181C & 0.002 & 0.305 \\
\hline P21 & Female & & GR & Sep 2004 & HSX & 0 & 905 & 699 & B & & G190A & & \\
\hline & & & & & & 10 & & & B & & G190A & 0.005 & 0.866 \\
\hline P22 & Male & & $G R$ & Jun 2004 & $?$ & 0 & 10500 & 918 & B & & G190A & & \\
\hline & & & & & & 13 & & & B & & G190A & 0.005 & 0.387 \\
\hline Tran & smitted & ariants harb & g onl & ated mutati & & & & & & & & & \\
\hline P23 & Male & & $\mathrm{NL}$ & Apr 2007 & HSX & 0 & 700000 & 664 & B & M46L & & & \\
\hline & & & & & & 14 & & & & M46L & & 0.000 & 1.000 \\
\hline & & & & & & 22 & & & & M46L & & 0.000 & 1.000 \\
\hline P24 & Male & Jan 2006 & $\mathrm{NL}$ & Apr 2008 & MSM & 0 & 5170 & 742 & B & M46L & & & \\
\hline & & & & & & 10 & & & & M46L & & 0.001 & 0.310 \\
\hline & & & & & & 29 & & & & M46L & & 0.004 & 0.471 \\
\hline P25 & Male & Jul 2005 & $\mathrm{NL}$ & Aug 2008 & MSM & 0 & 421000 & 409 & B & M46L & & & \\
\hline & & & & & & 14 & & & & M46L & & 0.000 & 1.000 \\
\hline & & & & & & 23 & & & & M46L & & 0.000 & 1.000 \\
\hline
\end{tabular}


Table 1 Patient characteristics, resistance mutations and evolution (Continued)

\begin{tabular}{|c|c|c|c|c|c|c|c|c|c|c|c|c|c|}
\hline \multirow[t]{3}{*}{ P26 } & male & & $\mathrm{NL}$ & Aug 2008 & MSM & 0 & 111000 & 657 & B & M46L & & & \\
\hline & & & & & & 14 & & & & M46L & & 0.000 & 1.000 \\
\hline & & & & & & 26 & & & & M46L & & 0.001 & 0.299 \\
\hline \multirow[t]{3}{*}{ P27 } & male & 05-11-04 & $\mathrm{NL}$ & Apr 2007 & MSM & 0 & 18100 & 699 & B & M46L & & & \\
\hline & & & & & & 13 & & & & M46L & & 0.000 & 1.000 \\
\hline & & & & & & 38 & & & & M46L & & 0.001 & 0.306 \\
\hline \multirow[t]{2}{*}{ P28 } & male & $15-02-03$ & $\mathrm{NL}$ & Mar 2005 & MSM & 0 & 69000 & 480 & B & L90M & & & \\
\hline & & & & & & 13 & & & & L90M & & 0.000 & 1.000 \\
\hline \multicolumn{14}{|c|}{ Transmitted variants harboring mutations against two drug classes } \\
\hline \multirow[t]{3}{*}{ P29 } & male & & $\mathrm{NL}$ & Dec 2001 & MSM & 0 & 288 & 1468 & B & & $\begin{array}{l}\text { D67G Y181CY } \\
\text { T215C K219E }\end{array}$ & & \\
\hline & & & & & & 10 & & & & & $\begin{array}{l}\text { D67G T215C } \\
\text { K219E }\end{array}$ & 0.006 & 0.148 \\
\hline & & & & & & 46 & & & & & $\begin{array}{l}\text { D67G T215C } \\
\text { K219E }\end{array}$ & 0.000 & 1.000 \\
\hline \multirow[t]{3}{*}{ P30 } & male & & $\mathrm{NL}$ & Jan 2005 & HSX & 0 & 26600 & 667 & B & G73S L90M & K103N & & \\
\hline & & & & & & 12 & & & & L90M & K103N & 0.001 & 0.306 \\
\hline & & & & & & 18 & & & & L90M & K103N & 0.001 & 0.306 \\
\hline \multirow[t]{2}{*}{ P31 } & female & & GR & Jul 2004 & HSX & 0 & 696 & 1288 & B & $\begin{array}{l}\text { I54V V82A } \\
\text { L90M }\end{array}$ & $\begin{array}{l}\text { M41L D67N } \\
\text { L210W T215D }\end{array}$ & & \\
\hline & & & & & & 10 & & & & $\begin{array}{l}\text { F53FL I54V } \\
\text { V82A L90M }\end{array}$ & $\begin{array}{l}\text { M41L D67N } \\
\text { L210W T215D }\end{array}$ & 0.001 & 0.293 \\
\hline
\end{tabular}

Abbreviations: $P R$ protease, $R T$ reverse transcriptase, NRTI nucleoside reverse transcriptase inhibitor, NNRTI non- nucleoside reverse transcriptase inhibitor, PI protease inhibitor, BE Belgium, GR Greece, NL the Netherlands, SL Slovenia, HSX heterosexual, MSM Men having sex with men? unknown route of transmission.

of 6 baseline polymorphisms in protease when compared to HXB2 (range: 4-9) and median of 5 when compared to consensus B (range: $3-8$ ).

\section{In vivo evolution of transmitted drug resistant HIV-1 variants}

The vast majority $(51 / 55)$ of TDRM persisted during the first year of follow-up. For $24 / 31$ patients a third and sometimes a fourth genotypic analysis was performed at a median of 28 months (range: 14-99 months) after the first sample. During this more extensive follow-up period of up to eight years, all resistance mutations present at one year after diagnosis persisted in the plasma (Table 1).

To gain more understanding of in vivo persistence of TDRM, we performed a comprehensive analysis of in vivo viral evolution during the follow-up. Viruses harboring protease drug-resistance mutations selected a median of 1 (range: 0-1) additional polymorphisms in protease during the first year of follow-up. Likewise, viruses harboring drug-resistance mutations in RT selected a median of 1 (range 0-3) additional RT polymorphisms (Table 2). As a measure of evolution at the nucleotide level, the p-distance between baseline and follow-up sequences was calculated. For the majority of patients, this revealed a very low $\mathrm{p}$-distance between baseline and one year, confirming limited viral evolution. In line with this observation, the $\mathrm{dN} / \mathrm{dS}$ ratio of the viral populations, which is an indicator of selection, did not change significantly in any patient (Table 2). However, in all transmitted viruses at least one change at a polymorphic site was observed, which is described in Table 2.

Impact of frequently observed TDRMs on in vitro RC

We determined the impact of TDRM on viral RC by introducing frequently observed drug-resistance mutations M46I, M46L or L90M in protease or M41L, M41L + $\mathrm{T} 215 \mathrm{Y}$ or $\mathrm{K} 103 \mathrm{~N}$ in $\mathrm{RT}$ in the background of the lab strain HXB2 by site-directed mutagenesis (Figure 1). Viruses were named according to mutations and origin; the prefix "SDM" indicates site-directed mutagenesis. The $\mathrm{RC}$ of all viral variants was determined in primary peripheral blood mononuclear cells (PBMCs), which are natural target cells for HIV. Site-directed mutants HIV-M184V, -I and $-\mathrm{T}$ with a known impact on $\mathrm{RC}$ were used as controls, and to enable comparison of $\mathrm{RC}$ between various experiments [27]. The difference in RC between HIVWT, $-\mathrm{M} 184 \mathrm{~V}$ and $-\mathrm{M} 184 \mathrm{I}$ has been demonstrated to be biologically relevant in vivo $[28,29]$.

All mutations caused a decrease in RC as compared to HIV-WT, except for mutation L90M in protease. The reduction in RC of the M41L, M41L + T215Y and K103N variants was comparable to each other, and to controls HIV-M184V and -I. M46I and M46L in protease resulted in the most severe reduction of RC (Figure 1). 
Table 2 Evolution of transmitted drug resistant HIV variants

\begin{tabular}{ccc}
\hline ID $\begin{array}{l}\text { Months } \\
\text { after } \\
\text { first } \\
\text { sample }\end{array}$ & $\begin{array}{l}\text { Protease amino } \\
\end{array}$ & Baseline \\
\hline Transmitted variants harboring o \\
P01 & 0 & S37N L63P 193L \\
& & \\
10 & \\
16 & \\
P02 & 0 & T12A K14KR \\
& & Q18HQ L191L \\
& & S37N L63P 193L
\end{tabular}

28

P03 0

P05 $0 \quad$ S37N 164V

99

21

P07 0

T12A |13V L19|

E35D S37D D60E I62V L63P A71T 172V 193L

L10I K14EV S37N L63T E65EV I72T V77I 193L

$115 \mathrm{~L}$ L19V S37N R41K D60E L63P I72IV 193L

$$
\begin{array}{ll}
-\mathrm{K} 14 \mathrm{KR}, & \mathrm{L} 19 \mathrm{~L}>\mathrm{IKLQ} \\
-\mathrm{Q} 18 \mathrm{HQ} & \\
\text { T12A }>\text { AT } & \text { L19IKLQ }>\text { IL }
\end{array}
$$
S37NS I64V C67CR
Reverse transcriptase amino acid 41-230

Baseline

Reversion

Additional mutations

M41L V60I I135T S162C K166R R211G L214F

$\begin{array}{ll}-\mathrm{K} 166 \mathrm{R} & +\mathrm{V} 106 \mathrm{IV} \\ & \mathrm{V} 106 \mathrm{IV}>\mathrm{I}\end{array}$

M41L V60I I135T

S162G K166EK I167F R211G L214F

$\begin{array}{ll}-S 162 \mathrm{G}-\mathrm{I167F} & \mathrm{K} 166 \mathrm{EK}>\mathrm{KR} \\ \mathrm{R} 211 \mathrm{G}>\mathrm{GR} & +\mathrm{T} 165 \mathrm{IT}\end{array}$

M41L V60I F61FS

E122K D123E 1178L V179IV E203EG Q207EQ L214F

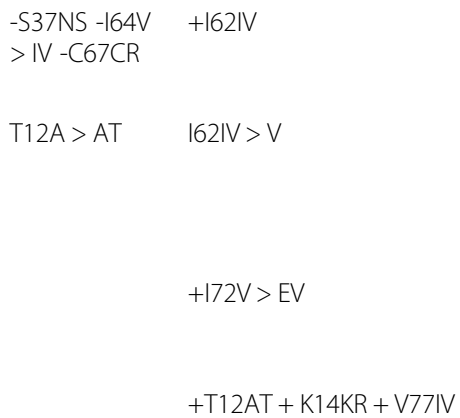

$+172 \mathrm{~V}>\mathrm{EV}$

$+\mathrm{T} 12 \mathrm{AT}+\mathrm{K} 14 \mathrm{KR}+\mathrm{V} 77 \mathrm{IV}$

K49R V60I V118I

E122K D123DE I135R S162D L210LS R211G

K64R R83K I178L I202V L214F T215D

$$
\begin{aligned}
& +\mathrm{M} 36 \mathrm{I} \\
& +\mid 13 I V+K 14 K R+K 45 K R
\end{aligned}
$$

-F61FS -E203EG

+S162X V179IV > I Q207EQ $>$ KQ + R211KR

$-S 162 X-1178 L-V 179 \mid$

Q207KQ $>$ EQ

\section{-L210LS D123DE $>E$ $\mathrm{S} 162 \mathrm{D}>\mathrm{S} 162 \mathrm{X}+$ T200IT + E204EK \\ $-S 162 X, R 211 G>G R \quad+T 165 I T$} -E204EK

$-K 64 R$ $+\mathrm{S} 68 \mathrm{~N}+\mathrm{E} 122 \mathrm{~K}$

$\mathrm{R} 83 \mathrm{~K}>\mathrm{KR},-1202 \mathrm{~V}$ $+\mathrm{A} 158 \mathrm{AS}+\mathrm{S} 162 \mathrm{~T}$

E122K I142V D177E Q207E L214F T215S

$\mathrm{K} 14 \mathrm{EV}>\mathrm{E}$ E65EV $>$ V

$-E 122 K>E K$

V60l S68G R83K V90I A98S E122P D123DEG 1135L S162C D177E I202IV R211K L214F T215D

$$
\begin{array}{ll}
-202 I V & \text { D123DEG }>\text { DE } \\
& \text { D123DE }>\text { DEG + } \\
& \text { T200IT }
\end{array}
$$

V60I S68G E122K

I135V S162NS T165IT

Q174HQ G196E

R211G L214F T215IT 
Table 2 Evolution of transmitted drug resistant HIV variants (Continued)

\begin{tabular}{|c|c|c|c|c|c|c|c|}
\hline P09 & 0 & $\begin{array}{l}\text { S37N I62V L63T } \\
\text { I64L V77| }\end{array}$ & & & $\begin{array}{l}\text { S68T E122K I135V } \\
\text { T139A G196E Q197R } \\
\text { L214F T215AT }\end{array}$ & & \\
\hline & 13 & & & & & $-\mathrm{T} 139 \mathrm{~A}$ & $+\mathrm{H} 198 \mathrm{HR}$ \\
\hline & 20 & & & $+\mathrm{R} 57 \mathrm{KR}$ & & $-\mathrm{H} 198 \mathrm{HR}$ & $\begin{array}{l}\text { S68T }>\text { AT }+ \\
\text { T139AT }+ \\
\text { T215AT }>\text { A }\end{array}$ \\
\hline P10 & 0 & $\begin{array}{l}\text { I15V E35D S37D } \\
\text { D60E L63P V77I } \\
\text { 193L }\end{array}$ & & & $\begin{array}{l}\text { S68K T69N A98S } \\
\text { L100LV E122K D123E } \\
\text { I135R N136NT Q145E } \\
\text { S162C I178M E194D } \\
\text { I195L G196E T200A } \\
\text { I202V Q207K R211G } \\
\text { L214F K219N H221Y } \\
\text { K223Q }\end{array}$ & & \\
\hline & 11 & & & $+\mathrm{R} 41 \mathrm{~K}$ & & -L100LV -N136NT & \\
\hline & 44 & & & $+K 45 K R+R 57 K R$ & & $-1135 R$ & $+K 49 K R$ \\
\hline P11 & 0 & $\begin{array}{l}\text { S37H R41KR R57K } \\
\text { Q61D }\end{array}$ & & & $\begin{array}{l}\text { V60I D67N T69E } \\
\text { V106I D121Y I135T } \\
\text { S162C D177E G196E } \\
\text { E203D Q207E R211KR } \\
\text { L214F T215C }\end{array}$ & & \\
\hline & 13 & & & & & $-\mathrm{V} 106 \mathrm{I} \mathrm{L} 214 \mathrm{~F}>\mathrm{FL}$ & $+\mathrm{T} 200 \mathrm{IT}$ \\
\hline P12 & 0 & $\begin{array}{l}\text { L10I T12S L19| } \\
\text { L63T }\end{array}$ & & & $\begin{array}{l}\text { V60I D67G S68G } \\
\text { I135T I178M R2111KR } \\
\text { L214F T215C K219E }\end{array}$ & & \\
\hline & 11 & & $-\mathrm{L} 10 \mathrm{l}$ & L19I > T & & $-R 211 K R$ & +E122EK \\
\hline & 24 & & & $+\mathrm{L} 10 \mathrm{~L}$ L19T > I + I62IV & & -E122EK -1135T & $\begin{array}{l}+\mathrm{Q} 207 \mathrm{LQR}+ \\
\text { R211KR }\end{array}$ \\
\hline P13 & 0 & $\begin{array}{l}\text { T12S L19| L63T } \\
\text { I64IM }\end{array}$ & & & $\begin{array}{l}\text { V60I D67G S68G } \\
\text { A158S I178M L214F } \\
\text { T215C K219E }\end{array}$ & & \\
\hline & 12 & & & $|64| M>M$ & & & $+\mathrm{E} 40 \mathrm{Q}$ \\
\hline & 14 & & & & & $-E 40 Q$ & \\
\hline P14 & 0 & T12S L19T L63T & & & $\begin{array}{l}\text { V60I D67G S68G } \\
\text { 1135IT I178M L214F } \\
\text { T215C K219E }\end{array}$ & & \\
\hline & 14 & & & & & & +E122EK I135IT > T \\
\hline P15 & 0 & $\begin{array}{l}\text { L19I E35D S37N } \\
\text { R57KR L63P V77IV } \\
\text { I93L }\end{array}$ & & & $\begin{array}{l}\text { M41LT69S D86DE } \\
\text { E122K S162C I178L } \\
\text { E204DE Q207EKQ } \\
\text { L210E R211K L214F } \\
\text { T215ST }\end{array}$ & & \\
\hline & 11 & & $-\mathrm{R} 57 \mathrm{KR}$ & V77IV $>1$ & & & $\begin{array}{l}\text { Q207EKQ > KQR } \\
\text { L210E > DE }\end{array}$ \\
\hline & 39 & & V77I > IV & $S 37 N>D N+R 57 K R$ & & & $\begin{array}{l}+V 60 V I+I 195 I L \\
Q 207 K Q R>X \\
L 210 D E>E \\
R 211 K>K N\end{array}$ \\
\hline & 77 & & '-R57KR & & & $S 162 C>C S$ & $\begin{array}{l}\text { E204DE > } \\
\text { DEKNR211KN > K }\end{array}$ \\
\hline P16 & 0 & $\begin{array}{l}\text { L19I E35D S37NS } \\
\text { L63P V77IV I93L }\end{array}$ & & & $\begin{array}{l}\text { M41L T69S D86E } \\
\text { K104KR E122K S162C } \\
\text { I178L E204DE } \\
\text { Q207KQR L210E } \\
\text { R211DEKN } \\
\text { L214FT215S }\end{array}$ & & \\
\hline
\end{tabular}


Table 2 Evolution of transmitted drug resistant HIV variants (Continued)

\begin{tabular}{|c|c|c|c|c|c|c|c|}
\hline & 13 & & & I72IM & & $-K 104 K R$ & $\begin{array}{l}\text { T69S }>\text { AS } \\
\text { S162C }>\text { CW }\end{array}$ \\
\hline & 33 & & $-1721 \mathrm{M}$ & $\begin{array}{l}\text { E35D > DEKN S37NS > N V77IV } \\
>1\end{array}$ & & $-E 204 D E$ & $\begin{array}{l}\text { T69AS > S } \\
\text { S162CW > W + } \\
\text { E194DE Q207KQR } \\
>\text { R R211DEKN > D }\end{array}$ \\
\hline & 49 & & & E35DEKN > DE & & $-E 194 D E$ & $\begin{array}{l}\text { Q207R > QR } \\
\text { R211D > DEKN }\end{array}$ \\
\hline \multirow[t]{3}{*}{ P17 } & 1 & $\begin{array}{l}\text { E35D R41K L63P } \\
\text { I93L }\end{array}$ & & & $\begin{array}{l}\text { K103N E122K D123E } \\
\text { R211K L214F }\end{array}$ & & \\
\hline & 12 & & & & & & $\begin{array}{l}+\mathrm{K} 173 \mathrm{KT}+ \\
\mathrm{D} 177 \mathrm{DN}\end{array}$ \\
\hline & 30 & & & $+S 37 N$ & & $-\mathrm{K} 173 \mathrm{KT}$ & $\begin{array}{l}+\mathrm{Q} 174 \mathrm{HQ}+ \\
\text { Q207QR R211K> } \\
\text { KQ }\end{array}$ \\
\hline \multicolumn{8}{|c|}{ Transmitted variants harboring only NNRTI-related mutations } \\
\hline P18 & 0 & $\begin{array}{l}\text { L10IV I13IV I15IV } \\
\text { L19IL I62V L63PS } \\
\text { I64LV C67S V77I }\end{array}$ & & & $\begin{array}{l}\text { K64R K103N E122K } \\
\text { D123E K173EK } \\
\text { Q174QR V179I T200A } \\
\text { R211K L214F }\end{array}$ & & \\
\hline & 12 & & $-\mathrm{L} 19 \mid \mathrm{L}$ & L10IV > I L63PS > X I64LV > V & & -K173EK -Q174QR & +D177DN \\
\hline & 28 & & & & & & $+\mathrm{R} 72 \mathrm{RS}+\mathrm{Q} 174 \mathrm{QR}$ \\
\hline \multirow[t]{4}{*}{ P19 } & 0 & $\begin{array}{l}\text { L10I I15V S37T } \\
\text { R41K C67G G68E } \\
\text { H69R }\end{array}$ & & & $\begin{array}{l}\text { V60I K103Q E122K } \\
\text { D123E I142V R211K } \\
\text { L214F }\end{array}$ & & \\
\hline & 10 & & & & & & \\
\hline & 12 & & & & & & \\
\hline & 59 & & & $\mathrm{G} 68 \mathrm{E}>\mathrm{D}$ & & & + T200IT \\
\hline \multirow[t]{3}{*}{ P20 } & 0 & $\begin{array}{l}\text { T12N K14R S37N } \\
\text { R41KR I64V }\end{array}$ & & & $\begin{array}{l}\text { E122K D123E I135T } \\
\text { Y181C T200A I202V } \\
\text { R211K L214F }\end{array}$ & & \\
\hline & 11 & & $\mathrm{~S} 37 \mathrm{~N}>\mathrm{NS}$ & & & & \\
\hline & 49 & & $\begin{array}{l}\mathrm{K} 14 \mathrm{R}>\mathrm{KR} \\
-\mathrm{R} 41 \mathrm{KR}\end{array}$ & $+\mathrm{E} 35 \mathrm{D}$ S37NS $>\mathrm{N}+\mathrm{L63HQ}$ & & $\begin{array}{l}\text { E122K > EK I135T > } \\
\text { IT }\end{array}$ & $\mathrm{D} 123 \mathrm{E}>\mathrm{AE}$ \\
\hline \multirow[t]{2}{*}{ P21 } & 0 & $\begin{array}{l}\text { I13V S37NT L63P } \\
\text { A71AG }\end{array}$ & & & $\begin{array}{l}\text { I50N G51W P52A } \\
\text { V60IV R83K A98AG } \\
\text { K101H S105LS D177E } \\
\text { V179I G190A R211K } \\
\text { L214F }\end{array}$ & & \\
\hline & 10 & & $-A 71 A G$ & S37NT > NST & & $\begin{array}{l}-150 N-G 51 Q-P 52 A \\
-A 98 A G-S 105 L S\end{array}$ & $\begin{array}{l}\text { V60IV > I + E122K } \\
\text { K173EK }\end{array}$ \\
\hline P22 & 0 & $\begin{array}{l}\text { I13V M36T S37N } \\
\text { L63P }\end{array}$ & & & $\begin{array}{l}\text { S48Q R83KR K101H } \\
\text { D123DE D177E V179| } \\
\text { G190A L214F H235R }\end{array}$ & & \\
\hline & 13 & & & M36T > IMT & & $-\mathrm{S} 48 \mathrm{Q}-\mathrm{H} 235 \mathrm{R}$ & $\begin{array}{l}\text { D123DE > DEKN + } \\
\text { S162CS }\end{array}$ \\
\hline P23 & 0 & $\begin{array}{l}\text { S37N M46L D60E } \\
\text { I62V L63S I72V } \\
\text { V77I I93L }\end{array}$ & & & $\begin{array}{l}\text { K49R V60I V118I } \\
\text { I135R E169D R211G } \\
\text { L214F }\end{array}$ & & \\
\hline & 14 & & & & & & +F87FL + E204EK \\
\hline & 22 & & & & & $\begin{array}{l}\text { V60I > IV -F87FL- } \\
\text { E204EK }\end{array}$ & \\
\hline
\end{tabular}


Table 2 Evolution of transmitted drug resistant HIV variants (Continued)

\begin{tabular}{|c|c|c|c|c|c|c|c|}
\hline \multirow[t]{3}{*}{ P24 } & 0 & $\begin{array}{l}\text { E35DE S37N M46L } \\
\text { D60E I62V L63S 193L }\end{array}$ & & & $\begin{array}{l}\text { K49KR V60I V118I } \\
\text { E122K I135R R211G }\end{array}$ & & \\
\hline & 10 & & $-E 35 D E-193 L$ & $+\mathrm{K} 70 \mathrm{KR}$ & & $-K 49 K R$ & \\
\hline & 29 & & $-K 70 K R$ & & & & $+\mathrm{K} 104 \mathrm{KR}+\mathrm{S} 162 \mathrm{C}$ \\
\hline \multirow[t]{3}{*}{ P25 } & 0 & $\begin{array}{l}\text { E35D S37NM46L } \\
\text { D60E I62V L63S } \\
\text { 193L }\end{array}$ & & & $\begin{array}{l}\text { K49R V60I V118I } \\
\text { E122K I135R R211G }\end{array}$ & & \\
\hline & 14 & & & $+\mathrm{R} 41 \mathrm{KR}$ & & & +D123E \\
\hline & 23 & & & L63S > PS 193L > IL & & & $\begin{array}{l}\text { D123E > DEKN + } \\
\text { I178ILV }\end{array}$ \\
\hline \multicolumn{8}{|c|}{ Transmitted variants harboring only PI-related mutations } \\
\hline \multirow[t]{3}{*}{ P26 } & 0 & $\begin{array}{l}\text { E35D S37N M46L } \\
\text { D60E I62V L63S } \\
\text { I93L }\end{array}$ & & & $\begin{array}{l}\text { K49R V60I V118I } \\
\text { E122K D123DN I135R } \\
\text { R211G }\end{array}$ & & \\
\hline & 14 & & & $+\mathrm{L} 191 \mathrm{~L}$ & & -D123DN & +T165IT \\
\hline & 26 & & & $\mathrm{~L} 191 \mathrm{~L}>\mathrm{X}+\mathrm{A} 71 \mathrm{AV}$ & & & $\begin{array}{l}\text { T165IT > I+ } \\
\text { E204EK }\end{array}$ \\
\hline \multirow[t]{3}{*}{ P27 } & 0 & $\begin{array}{l}\text { E35D S37N M46L } \\
\text { D60E I62V L63S } \\
\text { I93L }\end{array}$ & & & $\begin{array}{l}\text { K49R V60I V118I } \\
\text { E122K I135R N136NT } \\
\text { S162NS I167IT R211G }\end{array}$ & & \\
\hline & 13 & & & L63S > APS & & $\begin{array}{l}\text {-N136NT -S162NS } \\
\text {-I167IT }\end{array}$ & \\
\hline & 38 & & $-E 35 D$ & L63APS > A & & & \\
\hline \multirow[t]{2}{*}{ P28 } & 0 & $\begin{array}{l}\text { L19T S37N L63P } \\
\text { L90M 193L }\end{array}$ & & & $\begin{array}{l}\text { E122KT200A L214F } \\
\text { K220X }\end{array}$ & & \\
\hline & 13 & & $S 37 N>N S$ & & & $-K 220 X$ & \\
\hline \multicolumn{8}{|c|}{ Transmitted variants harboring mutations against two drug classes } \\
\hline \multirow[t]{3}{*}{ P29 } & 0 & $\begin{array}{l}\text { T4IT T12S L19IV } \\
\text { L63X }\end{array}$ & & & $\begin{array}{l}\text { V60l D67G S68G } \\
\text { K70KR I178M } \\
\text { Y181CY L214F } \\
\text { T215C K219E }\end{array}$ & & \\
\hline & 10 & & $-\mathrm{T} 4 \mathrm{IT}$ & +L10I L19IV > I L63X>T & & $-K 70 K R-Y 181 C Y$ & $\begin{array}{l}+11351 T+E 204 E G \\
+R 211 K R\end{array}$ \\
\hline & 46 & & $-\mathrm{L} 10 \mathrm{l}$ & $\begin{array}{l}\text { T12S > PS + G16AG L19I > IV + } \\
\text { M36IM L63T > PT + I64IV }\end{array}$ & & $-E 204 E G-R 211 K R$ & $|135| T>T$ \\
\hline \multirow[t]{3}{*}{ P30 } & 0 & $\begin{array}{l}\text { L10I I13V I15V I62V } \\
\text { L63P G73S L90M }\end{array}$ & & & $\begin{array}{l}\text { V60l A98S K103N } \\
\text { D121Y D123E I135T } \\
\text { R172KR L214F }\end{array}$ & & \\
\hline & 12 & & $-G 73 S$ & & & I135T > IT -R172KR & \\
\hline & 18 & & & $+S 37 N S$ & & & +K102KR \\
\hline \multirow[t]{2}{*}{ P31 } & 0 & $\begin{array}{l}\text { L10I I15V K20R } \\
\text { E35D M36I S37N } \\
\text { I54V Q58E L63P } \\
\text { A71V V82A L90M }\end{array}$ & & & $\begin{array}{l}\text { M41L K43N V60I } \\
\text { D67N E122P I135T } \\
\text { E138A I142V L210W } \\
\text { R211M L214F T215D }\end{array}$ & & \\
\hline & 10 & & & +F53FL & & $-\mathrm{L} 214 \mathrm{~F}$ & $+\mathrm{T} 1391+|178| \mathrm{V}$ \\
\hline
\end{tabular}

Patient-derived sequences are compared to HXB2. Bold positions indicate positions related to drug resistance, italics indicate polymorphisms of HXB2 compared to consensus B.

\section{In vitro RC of patient-derived HIV-1 variants harboring} frequently observed TDRM

Subsequently, the RC of frequently observed TDRM was determined in their natural genetic background (Figure 1). We constructed recombinant viruses using patientderived protease containing M46L, M46I or L90M, or patient-derived n-terminus of RT containing $\mathrm{M} 41 \mathrm{~L}$ or K103N into HXB2. In addition, two more complex transmitted viruses were studied: a protease-variant containing I54V + V82A + L90M and an RT-variant carrying M41L + T69S + L210E + T215S. Patient-derived clones are indicated by the prefix "p", followed by the TDRM. 


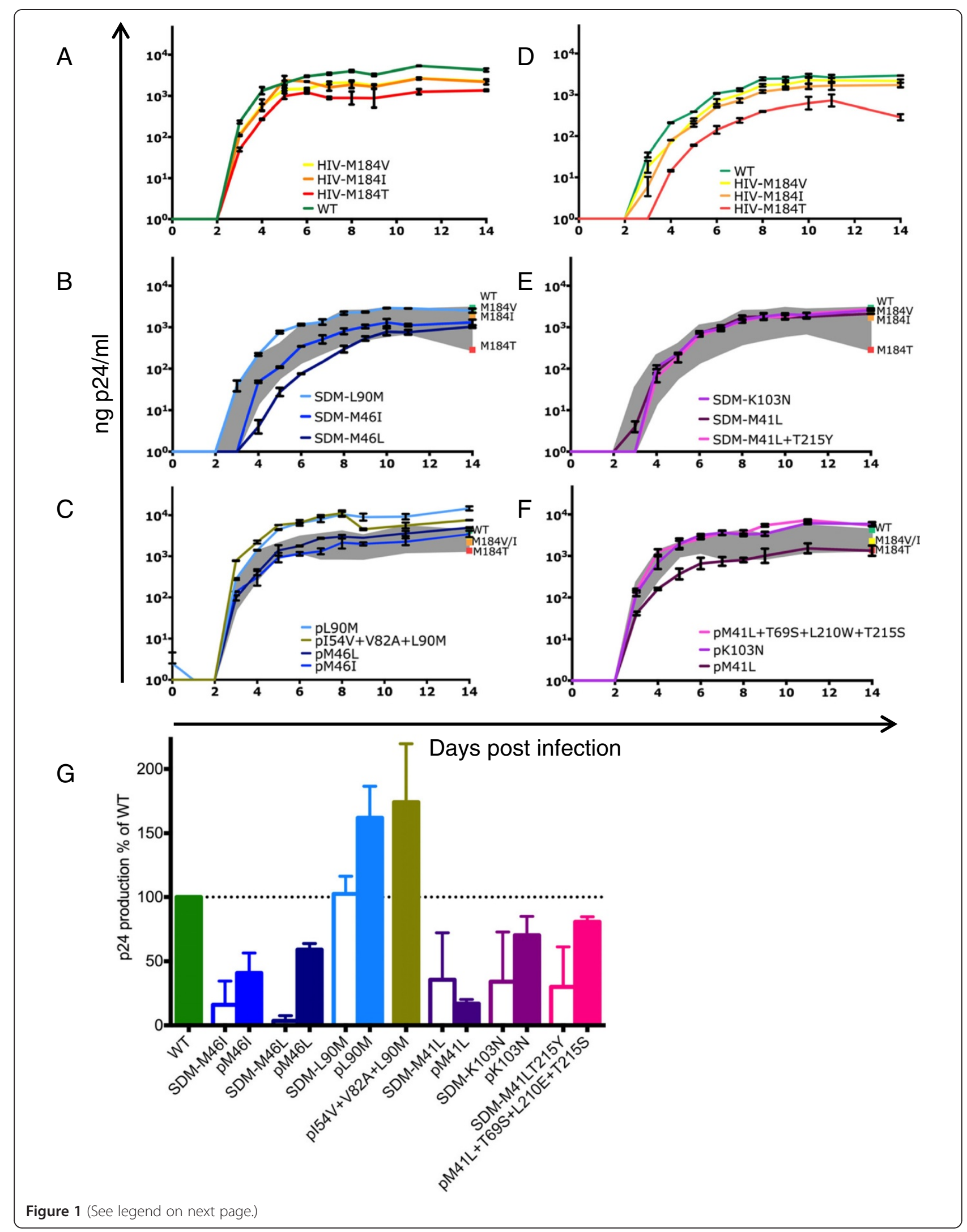


(See figure on previous page.)

Figure 1 Impact of frequently observed transmitted drug-resistance mutations on viral replicative capacity. The replicative capacity of site-directed mutant (SDM) viruses and patient-derived viruses was determined by infecting donor peripheral blood mononuclear cells with equal amounts of viral p24. In all experiments, control viruses HIV-M184V, -M184I and -M184T and wild type (WT) HIV were used as reference viruses. Representative experiments are shown in A-C and $\mathbf{D}-\mathbf{F}$. Error bars indicate standard deviation (SD) of mean within one experiment. Four biological replicates were performed for all viruses. (A-C) Replicative capacity of SDM-viruses (B, C) compared to control viruses (A). (D-F) Replicative capacity of patient-derived viruses (E, F) compared to control viruses (D). RC of WT and control viruses (A, D) is indicated in the corresponding graphs by a square, and the range in RC of WT and M184T by the grey area. (G) The median p24 production of both experiments as a percentage of WT in the corresponding experiment for all protease or reverse transcriptase mutant viruses. Error bars indicate range $(n=4)$.

The RC of p46I and p46L was similar to controls HIVM184I and $-\mathrm{V}$, indicating a diminished replication. The RT variant $\mathrm{pK} 103 \mathrm{~N}$ had an RC comparable to HIV-WT and the RC of pL90M was higher than HIV-WT. For M41L, it has been described that V60I and S162A function as compensatory mutations in transmitted HIV-1 variants [30]. We selected a patient-virus with M41L but without the potential compensatory mutations (pM41L). In this genetic background, the viral RC was as low as HIV-M184T and even lower than SDM-M41L. However, in vivo the variant containing this $\mathrm{M} 41 \mathrm{~L}$ mutation persisted for 8 months without selection of V60I or S162A before the patient initiated therapy (data not shown).

Interestingly, except for the pM41L variant, all patientderived viruses had a higher RC than the corresponding site-directed mutants (Figure 1). The RC of all protease mutation-harboring patient-derived viruses was higher than the corresponding SDM-viruses, and the RC of pL90M and pI54V + V82A + L90M were even higher than WT. In line with these results, the RC of pK103N and $\mathrm{pM} 41 \mathrm{~L}+\mathrm{T} 69 \mathrm{~S}+\mathrm{L} 210 \mathrm{E}+\mathrm{T} 215 \mathrm{~S}$ surpassed the RC of the corresponding SDM-viruses to the level of wild type virus. These observations suggest the presence of compensatory mutations in the genetic backbone of patient-derived viruses at the moment of diagnosis that are able to restore viral RC.

\section{Discussion}

In this study we strived to explain the in vivo persistence of the majority of TDRM in patients diagnosed with a drug-resistant HIV-1 variant. We selected patients diagnosed with HIV-1 containing limited profiles of TDRM, which are the most frequently transmitted variants as shown by large epidemiological studies [2,4]. In our patients, the vast majority of TDRM persisted for at least a year and up to eight years, confirming observations from previous studies that except for M184V/I, TDRM generally persist for longer than one year [10,13-25].

To explore the potential role of viral $\mathrm{RC}$ in persistence of TDRM, we investigated the impact of TDRM on the RC. In vitro determination of RC in PBMCs demonstrated that most site-directed mutant viruses harboring 1-2 frequently observed TDRMs had a reduced RC. However, in line with in vivo persistence the majority of patient-derived viruses had a higher RC than the corresponding SDM viruses. This suggests that polymorphisms, which may be present at baseline, can act as compensatory mutations. Our extensive sequence analysis demonstrated limited evolution on polymorphic positions, suggesting that in many transmitted HIV variants harboring TDRM compensatory mutations are already present at diagnosis.

Of the investigated site-directed mutant viruses, T215Y is known to evolve to atypical or partial revertant amino acids. Such alternative amino acids are known to confer limited impact on viral RC $[9,18,31]$, which is in line with the observed persistence of revertant and atypical T215 variants in our and other studies [10,13,15-25].

Interestingly, when present as a SDM in the commonly used lab strain HXB2, K103N decreased the RC in our experiments although this NNRTI-related mutation has been described to have a low impact in several [32-34] but not all [35] previous studies. This discrepancy may be due to the use of different assays or differences in replication caused by polymorphisms in lab strains. Indeed, the RC of patient-derived $\mathrm{K} 103 \mathrm{~N}$ was similar to WT virus, indicating that polymorphisms can restore viral RC. This may explain the in vivo persistence of $\mathrm{K} 103 \mathrm{~N}$ in our and previous studies $[10,21]$.

Several papers have described the impact of some drug resistance mutations on the RC of HIV-1 [16,32,33,35]. To our knowledge, the viral RC of frequently observed protease and RT TDRM has never been compared. Our data reveal that site-directed mutations at position 46 in protease have the most severe impact on RC.

Lack of reversion of the TDRM could be explained by a relatively small viral population size resulting in limited evolution. However, the median plasma HIV-RNA level of the included patients is similar to the HIV-RNA generally observed for newly diagnosed patients in the SPREAD programme [3]. Furthermore, although viral evolution was limited, in all transmitted viral variants changes at polymorphic sites were observed, indicating that replication could result in molecular evolution.

Certain resistance mutations such as M46I in protease have been described to decrease recognition of epitopes by certain HLA types [36]. As a result, also the immune system may affect viral evolution and persistence of TDRM. However, the majority of frequently observed TDRM may 
not impact or can even enhance recognition of epitopes $[36,37]$ and as such, it is unlikely that the immune system is the major driving force behind persistence of all TDRM.

We previously hypothesized based on an extensive literature study that the lack of reversion is related to the RC of transmitted HIV-1 variants harboring TDRM [9]. The currently described data confirms that TDRM may persist due to a high RC of the transmitted HIV-1 variant. Alternatively, the selection of additional mutations may restore the $\mathrm{RC}$ or result in compensatory fixation $[30,38]$. This important role of polymorphisms was supported by the differential impact of TDRM in the presence of patient-derived genetic background compared to site-directed mutants. For all but one investigated frequently observed TDRM, in vitro $\mathrm{RC}$ of patient-derived virus was higher than the corresponding SDM. A striking example is M46L. Although the single presence of M46L in HXB2 causes a large decrease in viral RC, this defect in RC is largely restored when M46L is present in a patient-derived genetic background.

M41L is one of the most frequently observed TDRM, and is an intriguing example emphasizing the impact of the genetic background on RC. As a single mutation, M41L in the background of wild type virus HXB2 decreased the $\mathrm{RC}$. This decrease was even more pronounced in the genetic background of pM41L, which was specifically selected for this study because of the absence of known compensatory mutations V60I and S162A [30]. In sharp contrast, $\mathrm{pM} 41 \mathrm{~L}+\mathrm{T} 69 \mathrm{~S}+\mathrm{L} 210 \mathrm{E}+\mathrm{T} 215 \mathrm{~S}$, the patient-derived virus with an extensive profile containing a M41L in the presence of the compensatory mutation V60I had a similar RC as wild type virus [30].

In addition, compensatory mutations may be observed outside the target gene of the antiviral compound. It has been demonstrated that mutations in gag may help to compensate the reduced protease activity conferred by resistance mutations in the protease itself [39]. Unfortunately sequencing of gag is usually not included in routine genotyping within Europe, impeding investigation of a potentially compensatory role of gag in this study. For $\mathrm{RT}$, compensatory mutations may also be present in the connection domain [40], which again is not included in routine genoptyping.

For only a subset of patients we had laboratory evidence of recent infection. We cannot exclude that patients were initially infected with a viral variant harboring a more extensive resistance profile and that some of these mutations had reverted before the patients were diagnosed. As such, the observed limited evolution of pol may be a result of viral adaptation before diagnosis or may even have taken place in previous hosts. By using a more sensitive sequence method, we might have been able to increase the detection of TDRM in the included patients [11]. However, we have previously used ultra-deep sequencing to investigate the quasispecies in plasma of patients who were newly diagnosed with an HIV-1 variant harboring a single NRTI-related resistance mutation. In most patients we were unable to detect viral minority variants harboring more extensive resistance profiles in the plasma, which may be suggestive of infection with a circulating HIV-1 variant harboring a limited resistance profile [41]. It is not unlikely that onward transmission of highly stable HIV-1 variants harboring limited resistance profiles greatly contributes to the current epidemic of transmitted drug resistant HIV-1 variants. Indeed, phylogenetic studies have demonstrated that onward transmission by untreated patients is a major source of transmission of drug-resistant HIV-1 [42-44].

It is of great clinical importance to be able to distinguish whether transmitted drug resistant HIV-1 variants harbor complex but partially reverted resistance profiles or circulating HIV-1 variants containing limited resistance profiles. For the frequently observed NNRTI-resistance mutation $\mathrm{K} 103 \mathrm{~N}$, it is well-known that it causes high levels of resistance against all first generation NNRTIs $[45,46]$. Even when $\mathrm{K} 103 \mathrm{~N}$ is present as minority variant, it can contribute to therapy failure [11]. Fortunately, the recently approved second-generation NNRTIs remain active against HIV-1 harboring a single K103N [47,48]. In contrast, we have demonstrated that the NRTI-related M41L in RT has limited impact on selection of resistance against currently used NRTIs [49]. M46I/L or L90M as a single TDRM in protease may cause low level resistance to commonly used protease inhibitors such as lopinavir.

\section{Conclusion}

In conclusion, we confirmed persistence of the most frequently observed TDRM. All transmitted HIV-1 variants harbored additional polymorphisms, with limited selection of additional mutations. Limited reversion of TDRM is in concordance with the high in vitro $\mathrm{RC}$ of patient-derived viruses harboring TDRM. As SDM viruses with the same TDRM as patient-derived viruses have a lower RC in vitro, we propose that polymorphisms that function as compensatory mutations (partially) restoring viral RC explain the in vivo persistence of TDRM. The stability of transmitted drug resistant HIV-1 variants can facilitate onward transmission of these viruses.

\section{Methods}

\section{In vivo evolution}

\section{Ethics statement}

Ethical requirements differ between countries according to national legislation. In countries where a national surveillance system was established, legally no informed consent was needed. In other countries, approval was obtained by the institutional medical ethical review committees. All data were anonymized at national level. 


\section{Patients}

Patients from four countries participating in the SPREADprogramme (Belgium, Greece, the Netherlands, Slovenia) were included. For all included patients, a baseline genotypic resistance test performed on a plasma sample within three months after diagnosis of HIV-1 infection revealed at least one mutation on a position associated with transmitted drug resistance as described in the mutation list as recommended by the WHO [26]. Patients were included on the basis of sample availability; a base line sample and a sample one year (10-14 months) later. If available, a sample at later time points were included. All included patients were at least 18 years of age and not exposed to antiretroviral therapy during the study period.

\section{Sequence analysis}

Genotypic resistance tests were performed by population sequencing of the viral protease and part of reverse transcriptase using commercially available assays or in-house methods covering at least amino acids 4-99 of protease and amino acids 30-249 of RT. All laboratories collaborated in the quality control program of ESAR to ensure high quality genotypic data [3,4]. HIV-1 subtype was determined using REGA 2.0 [50]. To investigate evolution, the p-distance and the ratio of the proportions of synonymous and nonsynonymous substitutions ( $\mathrm{dS} / \mathrm{dN}$ ratio) were calculated using MEGA 5.05. The p-distance is the proportion of nucleotides between two sequences that has been changed. The $\mathrm{dS} / \mathrm{dN}$ ratio, a measure of selection pressure [51], was calculated with the Nei-Gojobori method and statistically tested with a Z-test.

\section{In vitro determination of replicative capacity Virus panel}

Mutations M46I, M46L and L90M in protease and M41L, $\mathrm{M} 41 \mathrm{~L}+\mathrm{T} 215 \mathrm{Y}$ and $\mathrm{K} 103 \mathrm{~N}$ in $\mathrm{RT}$ were introduced in HXB2 by site-directed mutagenesis using the previously described vector systems CP-MUT and NRT-MUT [52] and the following primers: M46I 5'-GGA AAC CAA AAA TAA TAG GG-3' (HXB2 nucleotides 2380-2396), M46L 5' -GGA AAC CAA AAC TGA TAG GG-3' (HXB2 nucleotides 2380-2396), L90M 5'-GAA ATC TGA TGA CTC AGA TTG-3' (HXB2 nucleotides 2511-2532), M41L 5'-ATT TGT ACA GAG CTG GAA AAG GAA G-3' (HXB2 nucleotides 2658-2682), K103N 5'-GTT ACT GAT TTG TTC TTT TTT AAC CC-3' (HXB2 nucleotides 2844-2869), T215Y 5' -TGTCTG GTG TGTAAA GTCCCCACC-3' (HXB2 nucleotides 3181-3204).

Baseline patient-derived viral protease genes harboring M46I, M46L, L90M or I54V + V82A + L90M or the Nterminus of RT containing M41L, M41L + T69S + $\mathrm{L} 210 \mathrm{E}+\mathrm{T} 215 \mathrm{~S}$ or $\mathrm{K} 103 \mathrm{~N}$ were introduced into HXB2 using the same vector system [52].
Clones were obtained and sequence analysis was performed to verify resemblance to population sequences. Subsequently, at least three recombinant virus stocks were generated by Lipofectamine 2000 (Invitrogen) transfection of HEK293T cells according to manufacturer's guidelines. $\mathrm{TCID}_{50}$ was determined by end-point dilution in MT2 cells, demonstrating similar replication in this T cell line in all cases. A random clone was selected and quantified by p24 ELISA (Aalto Bioreagent, Dublin, Ireland) for the RC analysis.

\section{RC analysis}

PBMCs were isolated from HIV-seronegative blood donors by Ficoll-Paque density gradient centrifugation and stored in liquid nitrogen until use. To minimize differences between batches caused by variation between donors, each batch of PBMCs consisted of five combined donors. The $\mathrm{RC}$ of the virus panel was determined by infecting $5 \times 10^{6}$ phytohaemagglutinin-stimulated $(2 \mathrm{mg} / \mathrm{L})$ donor PBMCs with the equivalent of $40 \mathrm{ng}$ HIV-1 p24 for two hours. Subsequently, cells were washed twice and maintained for 14 days in RPMI1640 with L-glutamine (BioWhittaker), $10 \%$ fetal bovine serum (Biochrom AG), $10 \mathrm{mg} / \mathrm{L}$ gentamicin (Gibco) and $5 \mathrm{U} / \mathrm{ml} \mathrm{IL-2.} \mathrm{Cell-free} \mathrm{supernatant} \mathrm{was}$ harvested daily for monitoring of the $\mathrm{p} 24$ production. The $\mathrm{RC}$ of either the SDM-viruses or the patient-derived viruses was compared to the RC of control viruses (WT, HIV-M184V, -M184I and -M184T). By comparing viruses containing only the mutation(s) or gene of interest in the exact same HIV-WT background, it is possible to determine the impact of these relevant mutation(s) or genes on viral RC. For all viruses, replication curves were performed in four biological replicates divided over two independent experiments. The mean p24 production of two replicates within representative experiments are indicated in Figure $1 \mathrm{~A}-\mathrm{C}$ for protease and $1 \mathrm{D}-\mathrm{F}$ for RT. Figure $1 \mathrm{G}$ represents the median $\mathrm{p} 24$ production relative to HIV-WT of all four replicates on day 7 post infection.

\section{Competing interests}

The authors declare that they have no competing interests.

\section{Authors' contributions}

AW, MN and CB designed the study and laboratory experiments. MPi and DJ conducted the experiments and analysis. AW, KF, AB, AH, EM, DP, ML and MPo contributed clinical and virological data. MPi, AW, MN and CB wrote the manuscript. All co-authors contributed to the interpretation of the results, and have read and approved the final manuscript.

\section{Acknowledgements}

This work was supported by the Aids Fonds Netherlands [grant 2007034] and the European Community's Seventh Framework Programme (FP7/2007-2013) under the project "Collaborative HIV and Anti-HIV Drug Resistance Network (CHAIN)" [grant agreement $n^{\circ} 223131$ ].

We acknowledge the support of contributors to the SPREAD surveillance programme and members of the European Society for Antiviral Resistance (ESAR). We thank Petra van Ham and Leander Theissen for technical assistance, Daniel Struck from the ESAR-team in Luxembourg for assistance in data management and Antoinet van Kessel for assistance in project coordination. 


\section{Author details}

'Department of Virology, Viroscience Lab, Erasmus MC, Postbus 2040, 3000 CA Rotterdam, the Netherlands. ${ }^{2}$ Virology, Department of Medical Microbiology, University Medical Center Utrecht, Utrecht, the Netherlands. ${ }^{3}$ Institute of Tropical Medicine, Antwerpen, Belgium. ${ }^{4}$ AIDS Reference Laboratory of the Vrije Universiteit Brussel, subunit Universitair Ziekenhuis Brussels, Brussels, Belgium. ${ }^{5}$ Internal Medicine and Infectious Diseases, University Medical Center Utrecht, Utrecht, the Netherlands. ${ }^{6}$ National Retrovirus Reference Center, Department of Hygiene, Epidemiology and Medical Statistics, Athens, Greece. ${ }^{7}$ Institute of Microbiology and Immunology, Faculty of Medicine, University of Ljubljana, Ljubljana, Slovenia.

Received: 25 March 2014 Accepted: 5 November 2014 Published online: 29 November 2014

\section{References}

1. Arts EJ, Hazuda DJ: HIV-1 antiretroviral drug therapy. Cold Spring Harb Perspect Med 2012, 2:a007161.

2. Wheeler WH, Ziebell RA, Zabina H, Pieniazek D, Prejean J, Bodnar UR, Mahle KC, Heneine W, Johnson JA, Hall HI: Prevalence of transmitted drug resistance associated mutations and HIV-1 subtypes in new HIV-1 diagnoses, U.S.-2006. AIDS 2010, 24:1203-1212.

3. Vercauteren J, Wensing AM, van de Vijver DA, Albert J, Balotta C, Hamouda O, Kucherer C, Struck D, Schmit JC, Asjo B, Bruckova M, Camacho RJ, Clotet B, Coughlan S, Grossman Z, Horban A, Korn K, Kostrikis L, Nielsen C, Paraskevis D, Poljak M, Puchhammer-Stöckl E, Riva C, Ruiz L, Salminen M, Schuurman R, Sonnerborg A, Stanekova D, Stanojevic M, Vandamme AM, et al: Transmission of drug-resistant HIV-1 is stabilizing in Europe. J Infect Dis 2009, 200:1503-1508.

4. SPREAD programme: Transmission of drug-resistant HIV-1 in Europe remains limited to single classes. AIDS 2008, 22:625-635.

5. SPREAD programme: Recent dynamics of transmitted drug resistance in Europe: SPREAD programme 2006-2007. In 9th European Workshop on HIV \& Hepatitis Treatment Strategies \& Antiviral Drug Resistance. Volume 2. Paphos, Cyprus: Reviews in Antiviral Therapy \& Infectious Diseases; 2011:15-16.

6. Johnson VA, Calvez V, Gunthard HF, Paredes R, Pillay D, Shafer RW, Wensing AM, Richman DD: Update of the drug resistance mutations in HIV-1: March 2013. Top Antivir Med 2013, 21:6-14.

7. Martinez-Picado J, Martinez MA: HIV-1 reverse transcriptase inhibitor resistance mutations and fitness: a view from the clinic and ex vivo. Virus Res 2008, 134:104-123.

8. Nijhuis M, van Maarseveen NM, Boucher CA: Antiviral resistance and impact on viral replication capacity: evolution of viruses under antiviral pressure occurs in three phases. Handb Exp Pharmacol 2009, 189:299-320.

9. Pingen M, Nijhuis M, de Bruijn JA, Boucher CA, Wensing AM: Evolutionary pathways of transmitted drug-resistant HIV-1. J Antimicrob Chemother 2011, 66:1467-1480.

10. Jain V, Sucupira MC, Bacchetti P, Hartogensis W, Diaz RS, Kallas EG, Janini LM, Liegler T, Pilcher CD, Grant RM, Cortes R, Deeks SG, Hecht FM: Differential persistence of transmitted HIV-1 drug resistance mutation classes. J Infect Dis 2011, 203:1174-1181.

11. Li JZ, Paredes R, Ribaudo HJ, Svarovskaia ES, Metzner KJ, Kozal MJ, Hullsiek KH, Balduin M, Jakobsen MR, Geretti AM, Thiebaut R, Ostergaard L, Masquelier B, Johnson JA, Miller MD, Kuritzkes DR: Low-frequency HIV-1 drug resistance mutations and risk of NNRTI-based antiretroviral treatment failure: a systematic review and pooled analysis. JAMA 2011, 305:1327-1335.

12. Wainberg MA, Moisi D, Oliveira M, Toni TD, Brenner BG: Transmission dynamics of the M184V drug resistance mutation in primary HIV infection. J Antimicrob Chemother 2011, 66:2346-2349.

13. Barbour JD, Hecht FM, Wrin T, Liegler TJ, Ramstead CA, Busch MP, Segal MR, Petropoulos CJ, Grant RM: Persistence of primary drug resistance among recently HIV-1 infected adults. AIDS 2004, 18:1683-1689.

14. Castro H, Pillay D, Cane P, Asboe D, Cambiano V, Phillips A, Dunn DT, for the UKCGOHIVDR: Persistence of transmitted HIV-1 drug resistance mutations. J Infect Dis 2013, 208(9):1459-1463.

15. Brenner B, Routy JP, Quan Y, Moisi D, Oliveira M, Turner D, Wainberg MA: Persistence of multidrug-resistant HIV-1 in primary infection leading to superinfection. AIDS 2004, 18:1653-1660.

16. Brenner BG, Routy JP, Petrella M, Moisi D, Oliveira M, Detorio M, Spira B, Essabag V, Conway B, Lalonde R, Sekaly RP, Wainberg MA: Persistence and fitness of multidrug-resistant human immunodeficiency virus type 1 acquired in primary infection. J Virol 2002, 76:1753-1761.

17. Chan KC, Galli RA, Montaner JS, Harrigan PR: Prolonged retention of drug resistance mutations and rapid disease progression in the absence of therapy after primary HIV infection. AIDS 2003, 17:1256-1258.

18. de Ronde A, van Dooren M, van Der Hoek L, Bouwhuis $D$, de Rooij E, van Gemen B, de Boer R, Goudsmit J: Establishment of new transmissible and drug-sensitive human immunodeficiency virus type 1 wild types due to transmission of nucleoside analogue-resistant virus. J Virol 2001, 75:595-602.

19. Delaugerre C, Morand-Joubert L, Chaix ML, Picard O, Marcelin AG, Schneider V, Krivine A, Compagnucci A, Katlama C, Girard PM, Calvez V: Persistence of multidrug-resistant HIV-1 without antiretroviral treatment 2 years after sexual transmission. Antivir Ther 2004, 9:415-421.

20. Ghosn J, Pellegrin I, Goujard C, Deveau C, Viard JP, Galimand J, Harzic M, Tamalet C, Meyer L, Rouzioux C, Chaix ML: HIV-1 resistant strains acquired at the time of primary infection massively fuel the cellular reservoir and persist for lengthy periods of time. AIDS 2006, 20:159-170

21. Little SJ, Frost SD, Wong JK, Smith DM, Pond SL, Ignacio CC, Parkin NT, Petropoulos CJ, Richman DD: Persistence of transmitted drug resistance among subjects with primary human immunodeficiency virus infection. J Virol 2008, 82:5510-5518.

22. Neifer S, Somogyi S, Schlote F, Berg T, Poggensee G, Kuecherer C: Persistence of a sexually transmitted highly resistant HIV-1: pol quasispecies evolution over 33 months in the absence of treatment. AIDS 2006, 20:2231-2233

23. Pao D, Andrady U, Clarke J, Dean G, Drake S, Fisher M, Green T, Kumar S, Murphy M, Tang A, Taylor S, White D, Underhill G, Pillay D, Cane P: Long-term persistence of primary genotypic resistance after HIV-1 seroconversion. J Acquir Immune Defic Syndr 2004, 37:1570-1573.

24. Polilli E, Di Masi F, Sozio F, Mazzotta E, Alterio L, Cosentino L, Clerico L, Perno CF, Parruti G: Sequential transmission and long-term persistence of an HIV strain partially resistant to protease inhibitors. New Microbio/ 2009, 32:205-208.

25. Yerly S, Rakik A, De Loes SK, Hirschel B, Descamps D, Brun-Vezinet F, Perrin L: Switch to unusual amino acids at codon 215 of the human immunodeficiency virus type 1 reverse transcriptase gene in seroconvertors infected with zidovudine-resistant variants. J Virol 1998, 72:3520-3523.

26. Bennett DE, Camacho RJ, Otelea D, Kuritzkes DR, Fleury H, Kiuchi M, Heneine W, Kantor R, Jordan MR, Schapiro JM, Vandamme AM, Sandstrom P, Boucher CA, van de Vijver D, Rhee SY, Liu TF, Pillay D, Shafer RW: Drug resistance mutations for surveillance of transmitted HIV-1 drug-resistance: 2009 update. PLoS One 2009, 4:e4724.

27. Back NK, Nijhuis M, Keulen W, Boucher CA, Oude Essink BO, van Kuilenburg AB, van Gennip AH, Berkhout B: Reduced replication of 3TC-resistant HIV-1 variants in primary cells due to a processivity defect of the reverse transcriptase enzyme. EMBO J 1996, 15:4040-4049.

28. Wainberg MA, Drosopoulos WC, Salomon H, Hsu M, Borkow G, Parniak M, Gu Z, Song Q, Manne J, Islam S, Castriota G, Prasad VR: Enhanced fidelity of 3TC-selected mutant HIV-1 reverse transcriptase. Science 1996, 271:1282-1285.

29. Schuurman $R$, Nijhuis $M$, van Leeuwen $R$, Schipper $P$, de Jong $D$, Collis $P$, Danner SA, Mulder J, Loveday C, Christopherson C, Kwok S, Sninsky J, Boucher CAB: Rapid changes in human immunodeficiency virus type 1 RNA load and appearance of drug-resistant virus populations in persons treated with lamivudine (3TC). J Infect Dis 1995, 171:1411-1419.

30. Huigen MC, Albert J, Lindstrom A, Ohlis A, Bratt G, de Graaf L, Nijhuis M, Boucher $C A B$ : Compensatory fixation explains long term persistence of the M41L in HIV-1 reverse transcriptase in a large transmission cluster. In 15th International HIV Drug Resistance Workshop: Basic Principles and Clinical Implications. Sitges, Spain: Antiviral Therapy; 2006:S113.

31. Garcia-Lerma JG, Nidtha S, Blumoff K, Weinstock H, Heneine W: Increased ability for selection of zidovudine resistance in a distinct class of wild-type HIV-1 from drug-naive persons. Proc Natl Acad Sci U S A 2001, 98:13907-13912.

32. Armstrong $\mathrm{KL}$, Lee $\mathrm{TH}$, Essex M: Replicative fitness costs of nonnucleoside reverse transcriptase inhibitor drug resistance mutations on HIV subtype C. Antimicrob Agents Chemother 2011, 55:2146-2153.

33. Collins JA, Thompson MG, Paintsil E, Ricketts M, Gedzior J, Alexander L: Competitive fitness of nevirapine-resistant human immunodeficiency virus type 1 mutants. J Virol 2004, 78:603-611. 
34. Xu HT, Oliveira M, Quan Y, Bar-Magen T, Wainberg MA: Differential impact of the HIV-1 non-nucleoside reverse transcriptase inhibitor mutations $\mathrm{K} 103 \mathrm{~N}$ and $\mathrm{M} 230 \mathrm{~L}$ on viral replication and enzyme function. J Antimicrob Chemother 2010, 65:2291-2299.

35. Cong ME, Heneine W, Garcia-Lerma JG: The fitness cost of mutations associated with human immunodeficiency virus type 1 drug resistance is modulated by mutational interactions. J Virol 2007, 81:3037-3041.

36. Mueller SM, Spriewald BM, Bergmann S, Eismann K, Leykauf M, Korn K, Walter H, Schmidt B, Arnold ML, Harrer EG, Kaiser R, Schweitzer F, Braun P, Reuter $\mathrm{S}$, Jaeger $\mathrm{H}$, Wolf E, Brockmever NH, Jansen K, Michalik C, Harrer T, German Competence Network for HIBV/AIDS: Influence of major HIV-1 protease inhibitor resistance mutations on CTL recognition. J Acquir Immune Defic Syndr 2011, 56:109-117.

37. Mason RD, Bowmer MI, Howley CM, Gallant M, Myers JC, Grant MD: Antiretroviral drug resistance mutations sustain or enhance $C T L$ recognition of common HIV-1 Pol epitopes. J Immunol 2004, 172:7212-7219.

38. van Maarseveen NM, Wensing AM, de Jong D, Taconis M, Borleffs JC, Boucher CA, Nijhuis M: Persistence of HIV-1 variants with multiple protease inhibitor (PI)-resistance mutations in the absence of PI therapy can be explained by compensatory fixation. J Infect Dis 2007, 195:399-409.

39. Clavel F, Mammano F: Role of Gag in HIV resistance to protease inhibitors. Viruses 2010, 2:1411-1426.

40. von Wyl V, Ehteshami M, Symons J, Burgisser P, Nijhuis M, Demeter LM, Yerly S, Boni J, Klimkait T, Schuurman R, Ledergerber B, Götte M, Günthard HF, Swiss HIV Cohort Study: Epidemiological and biological evidence for a compensatory effect of connection domain mutation N348I on M184V in HIV-1 reverse transcriptase. J Infect Dis 2010, 201:1054-1062

41. Pingen $M$, van der Ende $M$, Wensing $A$, El Barzouhi $A$, Simen B, Schutten $M$, Boucher C: Deep sequencing does not reveal additional transmitted mutations in patients diagnosed with HIV-1 variants with single nucleoside reverse transcriptase inhibitor resistance mutations. HIV Med 2012, 14(3):176-181.

42. Brenner BG, Roger M, Routy JP, Moisi D, Ntemgwa M, Matte C, Baril JG, Thomas R, Rouleau D, Bruneau J, Leblanc R, Legault M, Tremblay C, Charest H, Wainberg MA, Quebec Primary HIV Infection Study Group: High rates of forward transmission events after acute/early HIV-1 infection. J Infect Dis 2007, 195:951-959.

43. Yerly S, Vora S, Rizzardi P, Chave JP, Vernazza PL, Flepp M, Telenti A, Battegay M, Veuthey AL, Bru JP, Swiss HIV Cohort Study, Rickenbach M, Hirschel B, Perrin L: Acute HIV infection: impact on the spread of HIV and transmission of drug resistance. AIDS 2001, 15:2287-t.

44. Volz EM, lonides E, Romero-Severson EO, Brandt MG, Mokotoff E, Koopman JS: HIV-1 transmission during early infection in men who have sex with men: a phylodynamic analysis. PLoS Med 2013, 10:e1001568. discussion e1001568

45. Bacheler L, Jeffrey S, Hanna G, D'Aquila R, Wallace L, Logue K, Cordova B, Hertogs K, Larder B, Buckery R, Baker D, Gallagher K, Scarnati H, Tritch R, Rizzo C: Genotypic correlates of phenotypic resistance to efavirenz in virus isolates from patients failing nonnucleoside reverse transcriptase inhibitor therapy. J Virol 2001, 75:4999-5008.

46. Kuritzkes DR, Lalama CM, Ribaudo HJ, Marcial M, Meyer WA 3rd, Shikuma C, Johnson VA, Fiscus SA, D'Aquila RT, Schackman BR, Acosta EP, Gulick RM: Preexisting resistance to nonnucleoside reverse-transcriptase inhibitors predicts virologic failure of an efavirenz-based regimen in treatmentnaive HIV-1-infected subjects. J Infect Dis 2008, 197:867-870.

47. Anta L, Llibre JM, Poveda E, Blanco JL, Alvarez M, Perez-Elias MJ, Aguilera A Caballero E, Soriano V, de Mendoza C: Rilpivirine resistance mutations in HIV patients failing non-nucleoside reverse transcriptase inhibitor-based therapies. AIDS 2013, 27(1):81-85.

48. Azijn H, Tirry I, Vingerhoets J, de Bethune MP, Kraus G, Boven K, Jochmans D, Van Craenenbroeck E, Picchio G, Rimsky LT: TMC278, a next-generation nonnucleoside reverse transcriptase inhibitor (NNRTI), active against wild-type and NNRTI-resistant HIV-1. Antimicrob Agents Chemother 2010, 54:718-727.

49. Pingen $M$, Nijhuis $M$, Boucher $C A B$, Wensing AMJ: The frequently transmitted M41L mutation does not affect the in vitro selection of resistance against tenofovir and emtricitabine. In 6th International Workshop on HIV Transmission. Volume 7. Rome, Italy: Infectious Diseases \& Antiviral Therapy; 2011:20.
50. de Oliveira T, Deforche K, Cassol S, Salminen M, Paraskevis D, Seebregts C, Snoeck J, van Rensburg EJ, Wensing AM, van de Vijver DA, Boucher CA, Camacho R, Vandamme AM: An automated genotyping system for analysis of HIV-1 and other microbial sequences. Bioinformatics 2005, 21:3797-3800.

51. Nei M, Gojobori T: Simple methods for estimating the numbers of synonymous and nonsynonymous nucleotide substitutions. Mol Biol Evol 1986, 3:418-426.

52. van Maarseveen NM, Huigen MC, de Jong D, Smits AM, Boucher CA, Nijhuis $\mathrm{M}$ : A novel real-time PCR assay to determine relative replication capacity for HIV-1 protease variants and/or reverse transcriptase variants. J Virol Methods 2006, 133:185-194.

doi:10.1186/s12977-014-0105-9

Cite this article as: Pingen et al:: Persistence of frequently transmitted drug-resistant HIV-1 variants can be explained by high viral replication capacity. Retrovirology 2014 11:105.

\section{Submit your next manuscript to BioMed Central and take full advantage of:}

- Convenient online submission

- Thorough peer review

- No space constraints or color figure charges

- Immediate publication on acceptance

- Inclusion in PubMed, CAS, Scopus and Google Scholar

- Research which is freely available for redistribution 